\title{
The Influence of GPX1 Pro198Leu, CAT C262T and MnSOD Ala16Val Gene Polymorphisms on Susceptibility for Non-Hodgkin Lymphoma and Overall Survival Rate at Five Years from Diagnosis
}

\author{
Adriana-Stela Cosma, Cristina Radu, Alexandra Moldovan, Alina Bogliș, George Andrei Crauciuc,
} Emőke Horváth, Marcela Cândea, Florin Tripon*

University of Medicine, Pharmacy, Sciences and Technology of Târgu Mureș, Romania

\begin{abstract}
Objective: The aim of the current study was to investigate possible associations between catalase C262T (CAT C262T), glutathione peroxidase 1 Pro198Leu (GPX1 Pro198Leu), manganese superoxide dismutase Ala16Val (MnSOD Ala16Val) gene polymorphisms and non-Hodgkin Lymphoma risk (NHL) in a Romanian population and the five-year overall survival rate of the NHL patients. Methods: We included in this case-control study 406 individuals, divided into two groups: the control group $(n=315)$ and the patients group ( $\mathrm{n}=91)$. The DNA was extracted from peripheral blood and amplified using specific techniques. Results: The variant homozygous genotype of GPX1 Pro198Leu represents a risk factor for NHL development and no associations regarding the risk for NHL were found for MnSOD Ala16Val and CAT C262T gene polymorphisms. Two of the studied polymorphisms were associated with the overall survival rate thus: negative association regarding MnSOD Ala16Val, associated with higher overall survival rate and a positive one regarding CAT C262T, associated with lower overall survival rate. Conclusions: According to our results, the mentioned polymorphisms may be considered as susceptible markers of the five-year overall survival rate for NHL patients. Future studies with a larger number of patients are needed to confirm our results.
\end{abstract}

Keywords: NHL, GPX1, CAT, MnSOD polymorphism

Received 11 February 2019 / Accepted 14 March 2019

\section{Introduction}

Non-Hodgkin Lymphoma (NHL) is a heterogeneous type of blood cancer characterized, like all malignancies, by an abnormal cellular proliferation [1]. NHL represents the most common type of lymphomas (90\%) and may be classified into two groups, mature B-cell neoplasms and mature T-cell and natural killer (NK)-cell neoplasms [1,2]. One study conducted by Perry et al. demonstrated that the frequency of NHL subtypes is significantly different in some geographical regions. Also, the median age of patients with low-grade and high-grade of NHL in North America is significantly higher than in the Far East [3]. These findings suggest that differences between some etiological aspects and host risk factors are likely responsible, but for a better understanding of these differences, more epidemiological studies are needed $[3,4]$.

In Romania or Eastern and Central Europe, only a few studies are being involved in analyzing new risk factors for NHL and even fewer studies are addressing the role and the risk of different genetic variants [5,6]. The distribution of NHL subtypes in South-eastern Europe reported by Dotlic et al. in 2015 was: $91.1 \%$ of B-cell lymphomas and $8.9 \%$ of NK-cell lymphomas [7].

Chronic inflammation is a major risk factor which was reported to be connected to NHL, although the mecha-

* Correspondence to: Florin Tripon

E-mail: tripon.florin.2010@gmail.com nism underlying this connection is still ambiguous [8]. An aspect that could sustain this matter is the fact that neoplasia is known to be in some cases induced by oxidative stress caused by inflammation, which can generate reactive oxygen species (ROS) that lead to oxidative DNA damage [9]. In addition, ROS propagate pro-inflammatory cytokines, including interleukin-1 which stimulates B cells to produce antibodies that trigger a signal for $\mathrm{B}$-cell activation [10].

Furthermore, a number of studies highlighted that several diseases such as arthritis, systemic lupus erythematosus, and Sjögren's syndrome, characterized all by a chronic B-cell activated phenotype, have been associated with an increased risk of developing NHL $[11,12,13]$.

ROS can also be inactivated by anti-oxidant enzymes such as catalase (CAT), glutathione peroxidase $(G P X)$ and manganese superoxide dismutase $(M n S O D)$ which protect the cells from the negative effects of oxidative stress $[10,14]$.

According to SNPedia [15] and NCBI [16], there is a consistent number of single nucleotide polymorphisms (SNPs) of the human catalase gene. In the last few years, there has been a significant number of case-control and meta-analysis studies $[17,18]$ focusing on the CAT C262T polymorphism (substitution of Cytosine with Thymine in the 262 position of the promotor) involved in the progression of neoplasia, but more studies regarding NHL are still needed $[10,19]$. 
GPX1 is an additional antioxidant enzyme involved in the cell protection mechanisms against the ROS effects, reducing lipid hydroperoxides to their corresponding alcohols and free hydrogen peroxide to water. A specific polymorphism caused by the substitution of Proline (Pro) with Leucine (Leu) at position 198 (GPX1 Pro198Leu, rs1050450), has previously been reported to be associated with decreased enzyme activity and increased risk for developing cancer [20].

Manganese superoxide dismutase $(M n S O D)$, another antioxidant enzyme, protects the cells against oxidative stress. The superoxide radicals are eliminated after their conversion to water and oxygen [18]. MnSOD Ala16Val is one of the few polymorphisms previously investigated in NHL patients $[10,21]$ and correlated with enzyme activity and cancer risk $[18,20,22]$.

To the best of our knowledge, none of the published studies have evaluated CAT, GPX1 and MnSOD gene polymorphisms in patients with NHL in Central or Eastern Europe.

The aim of the current study was to investigate possible associations between CAT C262T, GPX1 Pro198Leu, $M n S O D$ Ala16Val gene polymorphisms and NHL risk in a Romanian population and their influence on the overall survival rate of NHL diagnosed patients.

\section{Method}

\section{Patients and Controls}

We conducted a population based case-control study, which consisted of 406 individuals from the same geographical area with a similar ethnical background (central region of Romania), divided into two groups: the control and the patients group. The patients group consisted of 91 adults with a confirmed diagnosis of NHL, admitted to the Hematology Clinics, of Emergency Clinical County Hospital of Târgu Mureş, Romania, between 2010 and 2016. Our control group included 315 healthy participants with no hematological malignancies and no history of cancer or other chronic diseases.

The approval of the present study was obtained from the Ethics Committee of the University of Medicine and Pharmacy of Târgu Mureş and was conducted according to the principles of the Declaration of Helsinki.

Clinical and histological diagnosis of NHL has been made according to the WHO standards [2], with the following distribution of NHL subtypes: 35 patients with Diffuse large B-cell lymphoma (DLBCL), 16 patients with Follicular lymphoma (FL), 16 patients with Marginal zone B-cell lymphoma (MBZL), 10 patients with Primary Non-Hodgkin Lymphoma (Pl), 7 with T-Cell Lymphoma, 2 with Mucosa-associated lymphoid tissue lymphomas (MALT), and 5 other types.

Clinical and laboratory data, such as histopathological classification of the NHL, the Eastern Cooperative Oncology Group Scale of Performance Status (ECOG), International Prognostic Index (IPI), Ann Arbor stage, treatment response, five-year survival rate, Lactate dehydrogenase (LDH) levels and extranodal sites were collected and analyzed from the patients' medical records.

\section{Genotyping Procedures}

Two milliliters of fresh peripheral blood were collected from patients and controls, at the time of their routine blood collection, in sterile tubes with Ethylenediaminetetraacetic acid (EDTA), for DNA extraction. DNA was isolated using the Quick-gDNA MiniPrep kits (ZymoResearch, USA) and Wizard Genomic DNA Purification kits (Promega, Madison, WI, USA) according to the manufacturer's instructions.

The DNA absorbance was verified by spectrophotometric quantification (BioSpectometer, Eppendorf, Germany). In order to analyze the genotypes of the mentioned SNPs, RFLP-PCR technique was performed using specific primers and FastDigest restriction enzymes, as previously reported [18, 23, 24].

\section{Statistical Analysis}

Data analysis was performed using GraphPad InStat software, MedCalc. Chi-square test and Fisher's exact test in order to compare the distribution of qualitative variables between cases and controls. Associations between genotypes, combined variant genotypes (homozygous and heterozygous), allele distribution among NHL patients and control groups were calculated as odds ratios (OR), recommended in case-control studies, with 95\% confidence intervals (CI) and a significance level of 0.05 .

\section{Results}

The distribution of allele's frequency and genotypes of the CAT C262T, GPX1 Pro198Leu, and MnSOD Ala16Val among NHL patients and controls are shown in Table I.

No significant association was found between the presence of variant alleles of CAT C262T and MnSOD Ala16 Val SNPs and NHL risk, but the variant homozygous genotype of GPX1 Pro198Leu represents a risk factor for NHL development ( $p$ value $=0.04)$.

Although the number of cases analyzed is relatively low, we have studied the combined variant genotypes. The results for the combined CATC262T, GPX1 Pro198Leu and $M n S O D$ Ala16Val genotypes and alleles among NHL patients and controls, are presented in Table II. In this case, no significant differences were observed between the presences of variant genotypes or variant alleles for all three investigated SNPs and NHL risk.

Furthermore, we evaluated the effect of these SNPs in detail, taking into account different risk factors such as gender, age, histological subtype of the lymphoma, survival rate, treatment response, $\mathrm{LDH}$ levels, and extranodal sites. Table III summarizes the clinical characteristics of NHL patients according to the genotypes. No differences were found regarding elevated LDH levels and variant genotypes/ alleles for the investigated SNPs ( $p$ value $>0.05$ ). 
Table I. Distribution of CAT C262T, GPX1 Pro198Leu and MnSOD Ala16Val genotypes and alleles among NHL patients and controls

\begin{tabular}{|c|c|c|c|c|c|}
\hline & $\begin{array}{c}\text { NHL } \\
\text { Patients }\end{array}$ & Controls & $p$ value & OR & $95 \% \mathrm{Cl}$ \\
\hline \multicolumn{6}{|l|}{ CAT C262T } \\
\hline $\mathrm{CC}$ & 54 & 165 & Reference & & \\
\hline CT & 32 & 130 & 0.26 & 0.75 & $0.45-1.23$ \\
\hline TT & 5 & 20 & 0.80 & 0.76 & $0.27-2.13$ \\
\hline $\mathrm{CC}+\mathrm{CT}$ & 37 & 150 & 0.28 & 0.75 & $0.46-1.21$ \\
\hline \multicolumn{6}{|l|}{ Allele } \\
\hline C & 140 & 460 & Reference & & \\
\hline $\mathrm{T}$ & 42 & 170 & 0.33 & 0.81 & $0.55-1.19$ \\
\hline \multicolumn{6}{|l|}{ GPX1 Pro198Leu } \\
\hline Pro/Pro & 2 & 26 & Reference & & \\
\hline Pro/Leu & 59 & 205 & 0.08 & 3.74 & $0.86-16.23$ \\
\hline Leu/Leu & 30 & 84 & 0.04 & 4.64 & $1.03-20.76$ \\
\hline Pro/Leu+Leu/Leu & 89 & 289 & 0.05 & 4.00 & $0.93-17.20$ \\
\hline \multicolumn{6}{|l|}{ Allele } \\
\hline Pro & 63 & 257 & Reference & & \\
\hline Leu & 119 & 373 & 0.14 & 1.30 & $0.92-1.83$ \\
\hline \multicolumn{6}{|l|}{ MnSOD Ala16Val } \\
\hline Ala/Ala & 7 & 33 & Reference & & \\
\hline $\mathrm{Ala} / \mathrm{Val}$ & 64 & 208 & 0.54 & 1.45 & $0.61-3.43$ \\
\hline $\mathrm{Val} / \mathrm{Val}$ & 20 & 74 & 0.81 & 1.27 & $0.49-3.3$ \\
\hline $\mathrm{Ala} / \mathrm{Val}+\mathrm{Val} / \mathrm{Val}$ & 84 & 282 & 0.55 & 1.4 & $0.59-3.29$ \\
\hline \multicolumn{6}{|l|}{ Allele } \\
\hline Ala & 78 & 274 & Reference & & \\
\hline Val & 104 & 356 & 0.93 & 1.02 & $0.73-1.43$ \\
\hline
\end{tabular}

Table II. Distribution of combined CAT C262T, GPX1 Pro198Leu and MnSOD Ala16Val genotypes and alleles among NHL patients and controls

\begin{tabular}{lccccc}
\hline & $\begin{array}{c}\text { NHL } \\
\text { Patients }\end{array}$ & Controls & p value & OR & 95\% Cl \\
\hline Combined genotypes & & & & & \\
CC+Pro/Pro+Ala/Ala & 1 & 5 & Reference & & \\
CT+Pro/Leu+Ala/Val & 12 & 83 & 0.57 & 0.72 & $0.07-6.73$ \\
TT+Leu/Leu+Val/Val & 1 & 1 & 0.46 & 5 & $0.14-166.73$ \\
CT or TT+ & & & & & \\
$\begin{array}{l}\text { Pro/Leu or Leu/Leu+ } \\
\text { Ala/Val or Val/Val }\end{array}$ & 37 & 128 & 1 & 1.44 & $0.16-12.76$ \\
Combined alleles & & & & & \\
C+Pro+Ala & 12 & 68 & Reference & & \\
T+Leu+Val & 14 & 58 & 0.52 & 1.36 & $0.586-3.191$ \\
\hline
\end{tabular}

In addition, we investigated the impact of these SNPs by the clinical manifestations such as Ann Arbor stage, ECOG or IPI. No associations were found between the presumed risk groups and variant genotypes of CAT C262T, GPX1 Pro198Leu, and $M n S O D$ Ala16Val gene polymorphisms (Table III).

Regarding the influence of allele's and genotypes in treatment response, no differences were noticed between NHL patients outcome and the investigated SNPs ( $p$ value $>0.05$ ) (Table III).

Moreover, we performed a comparison between different variant (heterozygous and homozygous) genotypes of CAT C262T, GPX1 Pro198Leu, and MnSOD Ala16Val, and the survival rate of our patients. We found that two of the studied polymorphisms were associated with the survival rate thus: negative association regarding $M n S O D$ Ala16Val, associated with a higher survival rate $(p$ value $=$ $0.031, \mathrm{OR}=0.15$, CI 95\% 0.028-0.078) and a positive association regarding CAT C262T, associated with a lower survival rate $(p$ value $=0.025$, OR $=3.763$, CI 95\% 1.208 11.721). The results for both heterozygous genotypes of $M n S O D$ and $C A T$ are statistically significant. While analyzing in detail, by allele frequency, both $M n S O D$ 16Ala variant allele $(p$ value $=0.33, \mathrm{OR}=0.73, \mathrm{CI} 95 \% 0.4-1.3)$ and CAT 262C variant allele ( $p$ value $=0.27, \mathrm{OR}=1.435$, CI 95\% 0.76-2.67) showed no association. The study was based on a relatively low number of carriers of the variant homozygous genotype, a fact that may have led to a non-statistically significant results in those cases. However, according to combined variant genotypes (heterozygous and homozygous- Dominant model), the results remained statistically significant, but only for CATC262T polymorphism.

Life expectancy of NHL cases according to the genotypes is graphically represented in Figure 1. The five year overall survival rate is calculated and described with $\mathrm{Ka}$ plan Meier curves, and is estimated to be: CAT C262T $(p$ value $=0.017) \mathrm{CC}-80 \%, \mathrm{CT}-43 \%$, TT $-100 \%$, GPX1 Pro198Leu ( $p$ value $=0.704)$ Leu/Leu $-82 \%$, Pro/ Leu $-60 \%$, Pro/Pro - 100\%, MnSOD Ala16Val $(p$ value < 0.0001) Ala/Ala 43\% Val/Ala 70\%, Val/Val 75\%.

No differences were found regarding clinical and demographical characteristics of patients and CAT, GPX1 and $M n S O D$ variant genotypes ( $p$ value $>0.05$ ) (Table III).

\section{Discussions}

Important advances in the understanding of the molecular pathogenesis of malignant lymphomas have been made in the last few years. Several unforeseen compounds referring to the oncogenic signaling courses are studied in different trials in patients diagnosed with lymphoma [25]. The evidence towards the oxidative stress involvement as an important contributor to cancer, producing inflammation and DNA damage, is still standing [22].

Diminished $M n S O D$ activity has been proved to be found in lymphoma tumors, as its increased activity induces apoptosis and suppresses tumorigenesis [22]. Furthermore, it has been showed that MnSOD2 Ala16Val, GPX1 Pro198Leu, and CATC262T SNPs decrease the enzymatic activity, and by inducing DNA damage, they also produce a high risk for developing cancer [26,27].

In the present study, we investigated the CAT C262T, GPX1 Pro198Leu and MnSOD2 Ala16Val SNPs on NHL patients in order to evaluate the associations between the mentioned SNPs and NHL risk, clinical characteristics, treatment response and overall survival rate in a Romanian population, from the country's central region.

The CAT C262T polymorphism has been intensively studied because the allelic $\mathrm{T}$ variant is associated with a decreased enzyme activity [28], resulting in a high level of ROS which maintains the possibility of developing some types of cancer.

In a recent meta-analysis [17] of several studies regarding different cancer types, including hematological ones, it 


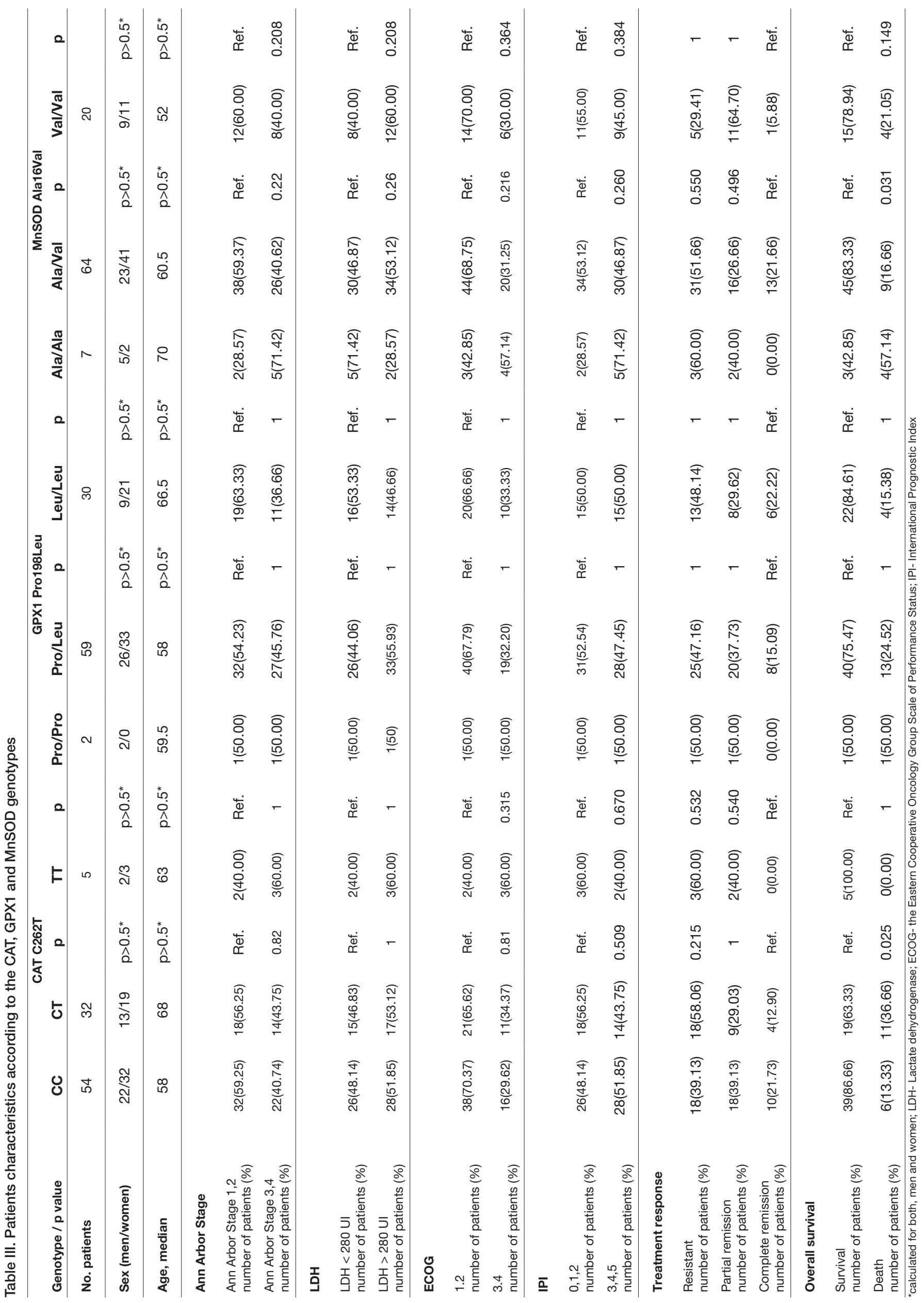



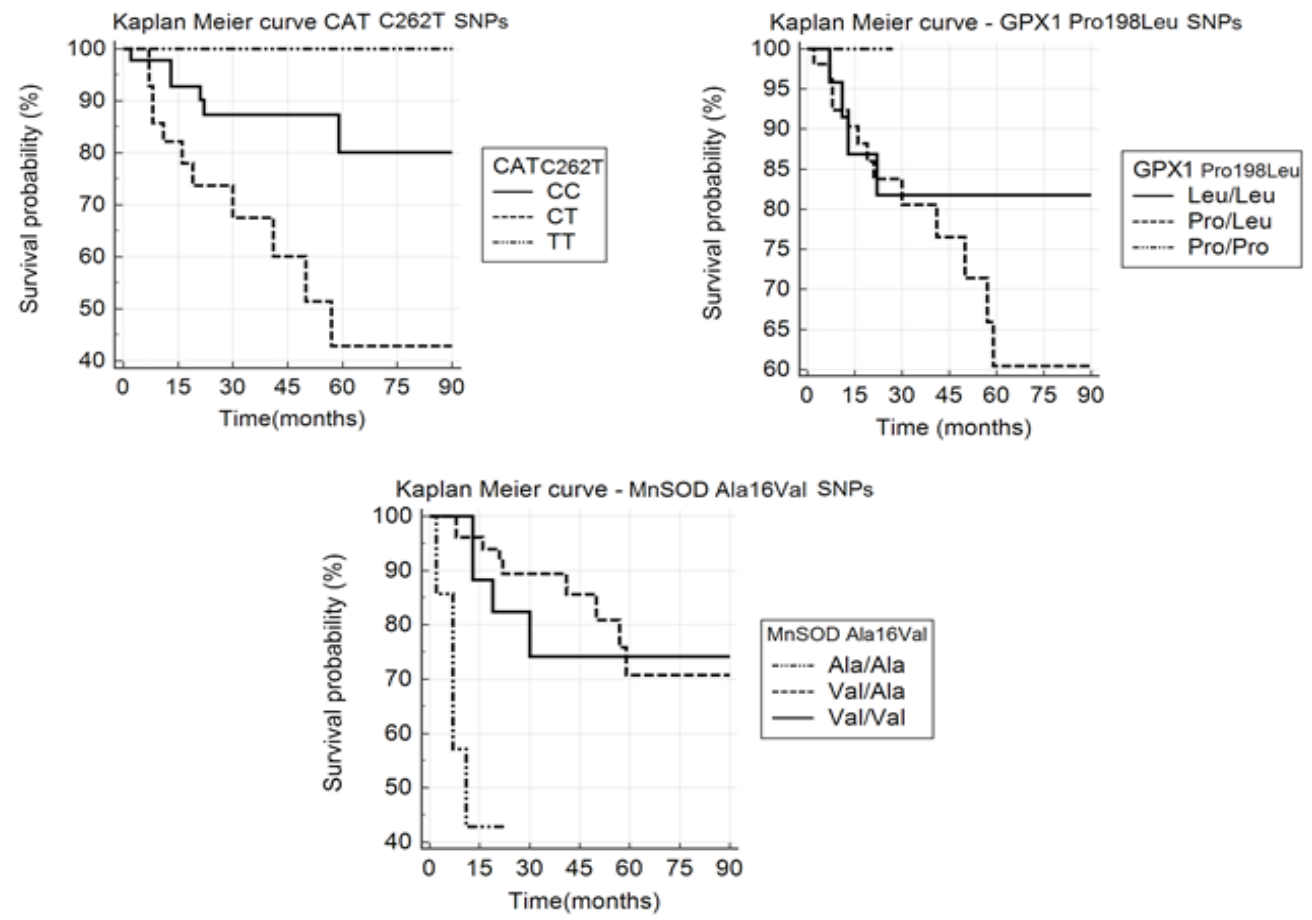

Fig. 1. Kaplan Meier survival curves of NHL patients according to the investigated CAT, GPX1 and MnSOD gene polymorphisms

was suggested that the CATC262T polymorphism may be associated with cancer susceptibility, and even more, may be considered a potential tumor biomarker. Nevertheless, the CAT C262T polymorphism is not considered an accurate prognostic factor for cancer survival rate [17].

According to our results, the CAT $\mathrm{C} 262 \mathrm{~T}$ is not a risk factor for NHL development, but it was associated with lower overall survival rate in dominant model. However, the results should be interpreted with caution, due to the relatively small number of patients.

Additionally, well designed, multicenter studies are required in order to investigate the role of this functional polymorphism, which should lead to a better and more comprehensive interpretation of the associations between the CAT C262T polymorphism and NHL risk and the overall survival rate, preferable on a wider population.

Paz-y-Mino et al. revealed in their study that the association of GPX1 Pro198Leu variant genotype with a decreased enzyme activity results in an increased risk of developing cancer in Caucasians [22]. Glutathione peroxidase has established its significant role as an antioxidant enzyme catalyzing the detoxification of hydrogen peroxide and being one of the most important enzymes discovered in humans [29].

In our study, we found an association between the variant homozygous genotype of GPX1 Pro198Leu and the susceptibility for NHL, the mentioned SNP being a risk factor for NHL developing. We found no statistically significant differences between clinical parameters, treatment response or survival rate and the GPX1 Pro198Leu polymorphism.

We found no differences in the distribution of the genotypes and alleles of MnSOD2 Val16Ala among the two groups, but we highlighted a negative association of the het- erozygous genotype and the survival rate, suggesting that the presence of this genotype may represent a protective factor, being associated with higher overall survival rate.

To our current knowledge, nowadays there is still no evidence supporting the role of these SNPs referring to the clinical characteristics as well as the five-year overall survival rate in the same treatment conditions and counting out other causes of death. Contrary to our findings, there are studies describing a significant association between $M n S O D$ Val6Ala polymorphism and the risk of developing DLB-NHL [21]. A meta-analysis performed by Kang et al. [30], showed an association between the $M n S O D$ Val16Ala and the NHL susceptibility and other types of cancer. In the current study, we discovered no association referring to this gene polymorphism and NHL subtypes ( $p$ value $>0.05$ ).

We found a similarity between the results of our analysis and a pooled analysis that investigated 2293 NHL cases and 3432 controls (from UK and USA) which found no association of this variant with the overall risk of developing NHL, but an association of the variant homozygous genotype with a decreased MZL risk was uncovered [10].

Superoxide dismutase has still an unclear role as its deficient activity has been highlighted in malignant lymphomas [31]. In some animal-based studies, it has been proved that a reduced $M n S O D$ activity leads to DNA damage and increased cancer incidence [32]. For that manner, further studies are needed in order to clarify the role of this gene.

The limitations of our study include the relatively small cohort of lymphoma patients and its retrospective design. The described impact of two additional polymorphisms in the manganese superoxidase dismutase and catalase gene on the overall survival in lymphoma is limited by the heterogeneous group of lymphoma diagnoses. Another limita- 
tion is represented by the lack of enzymatic level of catalase, glutathione peroxidase, superoxide dismutase.

\section{Conclusions}

In conclusion, the present study provides an evidence that the GPX1 Pro198Leu gene polymorphism might be a risk factor for NHL in a Romanian population and that there is a possible association between $C A T \mathrm{C} 262 \mathrm{~T}$ gene polymorphism and a low five-year overall survival rate in NHL patients. Furthermore, CAT C262T and MnSOD Val16Ala gene polymorphisms appear not to be risk factors for the development of NHL.

\section{Authors' contribution}

Adriana Stela Cosma (Conceptualization; Methodology; Project administration)

Cristina Radu, Alexandra Moldovan (Data curation)

Alina Bogliș (Formal analysis)

George Andrei Crauciuc (Formal analysis)

Emőke Horváth (Data curation; Formal analysis; Validation)

Marcela Cândea (Data curation; Formal analysis; Validation)

Florin Tripon (Conceptualization; Funding acquisition; Methodology; Resources; Supervision; Validation; Writing - review \& editing)

\section{Acknowledgement}

This work was supported by the University of Medicine and Pharmacy of Târgu Mureș, Romania, Research Grant number 15609/13/29.12.2017.

\section{Conflict of Interest}

The authors declare no conflict of interest.

\section{References}

1. Shankland KR, Armitage JO, Hancock BW. Non-Hodgkin lymphoma. Lancet. 2012; 380(9844):848-857.

2. Armitage JO, Gascoyne RD, Lunning MA, Cavalli F. Non-Hodgkin lymphoma. Lancet. 2017; 390(10091):298-310.

3. Perry AM, Diebold J, Nathwani BN et al. Non-Hodgkin lymphoma in the Far East: review of 730 cases from the International non-Hodgkin Lymphoma Classification Project. Ann Hematol. 2016; 95(2):245-251.

4. Perry AM, Diebold J, Nathwani BN et al. Non-Hodgkin Lymphoma In The Developing World: Review Of 4539 Cases From The International Non-Hodgkin Lymphoma Classification Project. Haematologica. 2016; 101(10):1244-1250.

5. Bogliș A, Radu CG, Tripon F et al. XRCC1 Arg194Trp and Arg399Gln Polymorphisms and Risk of Non-Hodgkin Lymphoma in a Romanian Population. Rev Med Chir Soc Med Nat lasi. 2016; 120(3):644-650.

6. Bogliș A, Crauciuc AG, Tripon F et al. No association between GSTT1, GSTM1 and GSTP1 gene polymorphism and risk of non-Hodgkin lymphoma in a population from Romania. International Journal of Innovation and Applied Studies. 2017; 19(1):1-8.

7. Dotlic S, Perry AM, Petrusevska G et al. Classification of non-Hodgkin lymphoma in South-eastern Europe: review of 632 cases from the international non-Hodgkin classification project. Br J Haematol. 2015; 171(3):366-372

8. Moriya K, Tamura H, Nakamura K, Hosone M, Inokuchi K. A primary esophageal MALT Iymphoma patient with Helicobacter pylori infection achieved complete remission after $\mathrm{H}$. pylori eradication without antilymphoma treatment. Leuk Res Rep. 2017; 7:2-5.
9. Reuter S, Gupta SC, Chaturvedi MM, Aggarwal BB. Oxidative stress, inflammation, and cancer: How are they linked? Free Radic Biol Med. 2010; 49 (11):1603-1616.

10. Lightfoot TJ, Skibola CF, Smith AG et al. Polymorphisms in the oxidative stress genes, superoxide dismutase, glutathione peroxidase and catalase and risk of non-Hodgkin's lymphoma. Haematologica. 2006; 91(9):1222-1227.

11. Baecklund $E$, lliadou $A$, Askling $J$ et al. Association of chronic inflammation, not its treatment, with increased lymphoma risk in rheumatoid arthritis. Arthritis Rheum. 2006; 54(3):692-701.

12. Callan MF. Epstein-Barr virus, arthritis, and the development of lymphoma in arthritis patients. Curr Opin Rheumatol. 2004; 16(4):399405.

13. Ramos-Casals M, De Vita S, Tzioufas AG. Hepatitis C virus, Sjogren's syndrome and B-cell lymphoma: linking infection, autoimmunity and cancer. Autoimmun Rev. 2005; 4(1):8-15.

14. Laurent A, Nicco C, Chéreau C et al. Controlling tumor growth by modulating endogenous production of reactive oxygen species. Cancer Res. 2005; 65(3):948-956.

15. SNPedia database. Catalase gene. Avaiable at https://www.snpedia. com/index. php?title=Special\%3ASearch\&search=catalase\&fulltext=1. Last time accessed on 27 January, 2019.

16. NCBI. Catalase gene. Available from http://ncbi.nlm.nih.gov/gene847. Last time accessed on 27 January, 2019.

17. Wang CD, Sun $Y$, Chen $N$ et al. The Role of Catalase C262T Gene Polymorphism in the Susceptibility and Survival of Cancers. Sci Rep. 2016; 6:26973.

18. Bănescu C, lancu M, Trifa AP et al. From Six Gene Polymorphisms of the Antioxidant System, Only GPX Pro198Leu and GSTP1 Ile105Val Modulate the Risk of Acute Myeloid Leukemia. Oxid Med Cell Longev. 2016; 6:1-10

19. Farawela $\mathrm{H}$, Khorshied M, Shaheen I et al. The association between hepatitis $\mathrm{C}$ virus infection, genetic polymorphisms of oxidative stress genes and B-cell non-Hodgkin's lymphoma risk in Egypt. Infect Genet Evol. 2012; 12(6):1189-1194.

20. Ekoue DN, Bera S, Ansong E et al. Allelic variations in MnSOD and GPX1 affect metabolism, mitochondrial membrane potential and expression of signaling proteins. Proceedings of the AACR 107th Annual Meeting 2016; April 16-20; New Orleans, LA: Cancer Res; 2016; 76(14).

21. Wang SS, David S, Cerhan JR et al. Polymorphisms in oxidative stress genes and risk for non-Hodgkin lymphoma. Carcinogenesis. 2006; 27(9):1828-1834.

22. Paz-y-Miño C, Muñoz MJ, López-Cortés A et al. Frequency of polymorphisms Pro198Leu in GPX-1 gene and lle58Thr in MnSOD gene in the altitude Ecuadorian population with bladder cancer. Oncol Res. 2010; 18(8):395-400.

23. Bănescu C, Trifa AP, Voidăzan S et al. CAT, GPX1, MnSOD, GSTM1, GSTT1, and GSTP1 genetic polymorphisms in chronic myeloid leukemia: a case-control study. Oxid Med Cell Longev. 2014; 2014: 875861.

24. Negovan A, lancu M, Tripon F, Crauciuc A, Mocan S, Bănescu C. The CAT-262 C>T, MnSOD Ala16Val, GPX1 Pro198Leu Polymorphisms Related to Oxidative Stress and the Presence of Gastric Lesions. J Gastrointestin Liver Dis. 2018; 27(4):371-378.

25. Nogai H, Dörken B, Lenz G. Pathogenesis of Non-Hodgkin's Lymphoma. J Clin Oncol. 2011; 29(14):1803-1811.

26. Al-Alem U, Gann PH, Dahl J et al. Associations between functional polymorphisms in antioxidant defense genes and urinary oxidative stress biomarkers in healthy, premenopausal women. Genes Nutr. 2012; 7(2):191-195.

27. Yuzhalin AE, Kutikhin AG. Inherited variations in the SOD and GPX gene families and cancer risk. Free Radic Res. 2012; 46(5):581-599.

28. Ahn J, Nowell S, McCann SE et al. Associations between catalase phenotype and genotype: modification by epidemiologic factors. Cancer Epidemiol Biomarkers Prev. 2006; 15(6):1217-1222.

29. Skibola CF, Curry JD, Nieters A. Genetic Susceptibility to Lymphoma. Haematologica. 2007; 92(7):960-969.

30. Kang SW. Superoxide dismutase 2 gene and cancer risk: evidence from an updated meta-analysis. Int J Clin Exp Med. 2015; 8(9):14647-14655.

31. Bewick M, Coutie W, Tudhope GR. Superoxide dismutase, glutathione peroxidase and catalase in the red cells of patients with malignant lymphoma. Br J Haematol. 1987; 65(3):347-350.

32. Van Remmen H, Ikeno Y, Hamilton M et al. Life-long reduction in MnSOD activity results in increased DNA damage and higher incidence of cancer but does not accelerate aging. Physiol Genomics. 2003; 16(1):29-37. 